\title{
A joint spectro-imaging analysis of the XMM-Newton and HESS observations of the supernova remnant RX J1713.7-3946
}

\author{
F. Acero ${ }^{1}$, J. Ballet ${ }^{1}$, A. Decourchelle ${ }^{1}$, M. Lemoine-Goumard ${ }^{2,3}$, M. Ortega ${ }^{4}$, E. Giacani ${ }^{4}$, \\ G. Dubner ${ }^{4}$, and G. Cassam-Chenai ${ }^{5}$ \\ ${ }^{1}$ Laboratoire AIM, CEA/DSM-CNRS-Université Paris Diderot, IRFU/SAp, CEA-Saclay, 91191 Gif-sur-Yvette, France \\ 2 CNRS/IN2P3, Centre d'Études Nucléaires de Bordeaux Gradignan, UMR 5797, Gradignan 33175, France \\ 3 Université Bordeaux I, Centre d'Études Nucléaires de Bordeaux Gradignan, UMR 5797, Gradignan 33175, France \\ 4 Instituto de Astronomía y Física del Espacio (IAFE), CC 67, Suc. 28, 1428 Buenos Aires, Argentina \\ INAF-Osservatorio Astrofisico di Arcetri, Largo E. Fermi, 5, 50125 Firenze, Italy
} e-mail: fabio.acero@cea.fr

Received 19 December 2008 / Accepted 31 May 2009

\section{ABSTRACT}

\begin{abstract}
Context. The supernova remnant (SNR) RX J1713.7-3946 (also known as G347.3-0.5) is part of the class of remnants dominated by synchrotron emission in X-rays. It is also one of the few shell-type SNRs observed at TeV energies allowing us to investigate particle acceleration at SNRs shock.

Aims. Our goal is to compare spatial and spectral properties of the remnant in $\mathrm{X}$ - and $\gamma$-rays to understand the nature of its TeV emission. This requires the study of the remnant on the same spatial scale at both energies. To complement the non-thermal spectrum of the remnant, we attempt to provide a reliable estimate of the radio flux density.

Methods. In radio, we revisited ATCA data and used HI and mid-infrared observations to differentiate between the thermal and the non-thermal emission. In X-rays, we produced a new mosaic of the remnant and degraded the spatial resolution of the X-ray data to the resolution of the HESS instrument to perform spatially resolved spectroscopy at the same spatial scale in X-and $\gamma$-rays. Radial profiles were obtained to investigate the extension of the emission at both energies.

Results. We found that part of the radio emission within the SNR contours is thermal in nature. Taking this into account, we provide new lower and upper limits to the integrated synchrotron flux of the remnant at $1.4 \mathrm{GHz}$, of $22 \mathrm{Jy}$ and $26 \mathrm{Jy}$, respectively. In X-rays, we obtained the first full coverage of RX J1713.7-3946 with XMM-Newton. The spatial variation in the photon index seen on small scale in X-rays is smeared out at HESS resolution. A non-linear correlation between the X-and $\gamma$-ray fluxes of the type $F_{X} \propto F_{\gamma}^{2.41}$ is found. If the flux variation is mainly caused by density variation around the remnant then a leptonic model can more easily reproduce the observed $\mathrm{X} / \gamma$-ray correlation. In some angular sectors, radial profiles indicate that the bulk of the X-ray emission comes more from the inside of the remnant than in $\gamma$-rays.
\end{abstract}

Key words. ISM: supernova remnants - X-rays: individuals: RX J1713.7-3946 - acceleration of particles radiation mechanisms: non-thermal

\section{Introduction}

Supernova remnants (SNRs) have long been believed to be accelerators of cosmic rays at least up to the knee $\left(\sim 10^{15} \mathrm{eV}\right)$. Evidence that electrons are indeed accelerated in SNRs is found both in radio and $\mathrm{X}$-rays through synchrotron emission. The detection of $\mathrm{TeV}$ emission from a SNR is evidence of proton acceleration if the $\gamma$-rays originate from the interaction of accelerated protons with the ambient matter (hadronic model). However, the $\gamma$-rays can also be produced by inverse Compton scattering of accelerated $\mathrm{TeV}$ electrons off ambient photons (leptonic model) that could be either infrared surrounding emission or cosmic microwave background. The SNRs interacting with dense molecular clouds are good candidates for detecting $\gamma$-ray emission from the hadronic mechanism because the high density of the cloud provides a large amount of targets for the accelerated protons. The SNR RX J1713.7-3946 (also known as G347.3-0.5) is one of those candidates because it is interacting with a dense molecular cloud both in the northwest (NW) and the southwest (SW) of the remnant (Fukui et al. 2003; Cassam-Chenaï et al. 2004; Moriguchi et al. 2005).
RX J1713.7-3946 was first discovered in X-rays with the ROSAT all-sky survey in 1996 (Pfeffermann \& Aschenbach 1996). The remnant is close to the Galactic plane and the value of its distance is controversial. Using ASCA observations and the measurement of the column density toward the source, the distance of $1 \mathrm{kpc}$ was first proposed by Koyama et al. (1997). Slane et al. (1999) then derived a distance of $6 \mathrm{kpc}$ based on a possible association of the remnant with molecular clouds and the HII region G347.611+0.204. The comparison between the $\mathrm{X}$-ray and the HI absorbing column densities (Cassam-Chenaï et al. 2004), as well as CO observations of a molecular cloud interacting with the remnant (Fukui et al. 2003) both suggest a closer distance of $1 \mathrm{kpc}$ (value adopted in this paper). In this case, the remnant is about $20 \mathrm{pc}$ in diameter $(70$ arcmin on the sky) and could be associated with the supernova that exploded in AD 393 in the tail of the constellation Scorpius (Wang et al. 1997). The remnant would then be about 1600 years old.

In the radio band, Slane et al. (1999) showed for the first time an image of RX J1713.7-3946, based on MOST observations at $843 \mathrm{MHz}$. Later on, Ellison et al. (2001) and Lazendic et al. (2004, with a recalibrated and improved image) reported ATCA 
radio observations of this $\mathrm{SNR}$ at $1.4 \mathrm{GHz}$ (left-hand panel of Fig. 5). The SNR appears as a faint nebula $\left(\sim 1^{\circ}\right.$ in diameter $)$ with many short, curved features, the brightest of which are the two bright arcs visible at the northwest corner. These arcs coincide with the edges of the brightest X-ray emission. Interestingly, those arcs are observed to be in the vicinity of the HII region G347.611+0.204, which is located at $6.6 \mathrm{kpc}$ (Russeil 2003; Conti \& Crowther 2004). An almost complete ring of weak emission, about 30 arcmin in size, can also be detected close to the center of this extended SNR. An accurate estimate of the integrated radio flux density of RX J1713.7-3946 is a pending problem. This is because of the intrinsic faintness of this SNR, the possible mixing of the SNR synchrotron emission with the thermal emission of the nearby HII region and the limited quality of the available data.

In X-rays, the emission is dominated by a non-thermal continuum and no emission lines have been observed so far (Koyama et al. 1997; Slane et al. 1999; Pannuti et al. 2003; Cassam-Chenaï et al. 2004). This non-detection can set an upper limit to the ionization age. Assuming $t_{\mathrm{SNR}}=1600 \mathrm{yr}$, an upper limit to the density of the ambient medium of $0.02 \mathrm{~cm}^{-3}$ is then derived (Cassam-Chenaï et al. 2004). The study of the remnant with XMM-Newton on small spatial scales carried out by Cassam-Chenaï et al. (2004) and Hiraga et al. (2005) clearly showed a spatial variation in the photon index. It varies from 1.9 to 2.6 with a mean statistical error of $4 \%$ (Cassam-Chenaï et al. 2004). A correlation between the X-ray flux and the photon index is also observed. Those results were obtained using an adaptive grid that had approximately the same number of counts in each pixel grid region, where a spectrum was then extracted. In the bright regions (the north-west rim), the size of the grid pixel was typically $0.05^{\circ}$.

In $\gamma$-rays, RX J1713.7-3946 was detected by the CANGAROO collaboration (Muraishi et al. 2000; Enomoto et al. 2002). It was then definitely established by the HESS telescopes which provided the first spatially resolved $\gamma$-ray image of the remnant (Aharonian et al. 2004). The overall morphology is very similar to that in X-rays (same western shell) and the brightest spots (located to the north-west) are coincident. Since the remnant is extended and is the brightest shell-type SNR seen in $\gamma$-rays, its emission can be studied in detail. With the good spatial resolution of the HESS telescopes $\left(\sim 0.1^{\circ}\right)$, it has been possible to carry out a spatially resolved spectral study on large scales (Aharonian et al. 2006, hereafter AH06). No evidence of spatial variations in the photon index from region to region (mean value of 2.09 and a standard deviation of 0.07 ) was found down to the precision of the measurements (mean $1 \sigma$ statistical error of \pm 0.08 ). Moreover, there does not seem to be any correlation between the $\gamma$-ray flux and the photon index (Fig. 14 of AH06).

Those $\gamma$-ray results differ from those found on small scales in X-rays. However, we note that the $\mathrm{X}$ - and $\gamma$-rays results were not obtained for the same spatial scales (the spectra were not extracted using the same size of extraction regions) and the point spread function of both instruments is also very different. Those differences prevent a reliable comparison of the results at both energies. We must determine whether the spectral parameters really differ between the $\mathrm{X}$ - and $\gamma$-rays, or if this difference is caused by both studies analyzing different spatial scales.

To investigate this question, we carried out a detailed comparison between the remnant in X-and $\gamma$-rays using the same extraction regions and taking into account the different spatial resolution of the two instruments. We compared both the spectral properties of the remnant and the morphology using radial profiles in $\mathrm{X}$ - and $\gamma$-rays.

Concerning the radio flux estimate, we tried to determine which part of the radio emission is synchrotron emission in origin (related to the remnant) or thermal emission (possibly related to the nearby HII region). We then calculated the integrated flux density by reanalyzing data presented in Lazendic et al. (2003).

In Sect. 2, we present the different sets of data used in this study including the new XMM-Newton observations that complete the mosaic of the remnant. Section 3 presents the methods used for the processing of the X-ray data. In Sect. 4, the estimate of the integrated radio flux of the remnant and the results of the $\mathrm{X}$ - and $\gamma$-ray spectral and morphological comparison are shown. In Sect. 5, we discuss this comparison in the framework of hadronic and leptonic model. The implications of the newly measured radio flux for the multiwavelength emission models of the remnant are also discussed.

\section{Data}

\subsection{Radio data}

We reanalyzed ATCA observations carried out at $1.4 \mathrm{GHz}$ (Lazendic et al. 2004) following different paths to estimate the radio flux density of RX J1713.7-3946. The details of how to carry out an accurate calculation are discussed in Sect. 4.1.

\subsection{Infrared data}

Infrared observations in and around the remnant can help to differentiate between the thermal and the non-thermal radio emission, which is crucial for estimating the radio flux of the entire remnant. We used a method based on a color-color criteria proposed by Reach et al. (2006) using Spitzer data at 3.6, 4.5, 5.8, and $8 \mu \mathrm{m}$ from the Galactic Legacy Infrared Mid-Plane Survey Extraordinaire (GLIMPSE, Benjamin et al. 2003). The spatial resolution of the survey is about 2 arcsec. More details can be found in Sect. 4.1.

\subsection{XMM-Newton data}

The first set of observations of the SNR RX J1713.7-3946 (presented in Cassam-Chenaï et al. 2004) covered almost the entire remnant. New observations of the south, east and west regions completed the mosaic of the remnant. With a total of 11 pointings (see Table 1) we can now provide the first full mapping of RX J1713.7-3946 with XMM-Newton (see Fig. 1).

To remove proton flare contamination from the event files, we compiled a histogram of counts/seconds in the 10-12 keV band for the MOS cameras (12-14 keV for PN). We then fitted a Gaussian distribution to the histogram and considered, in the observation, only the time intervals where the count rates are within a $3 \sigma$ range (Pratt \& Arnaud 2002). Table 1 provides the list of the remaining exposure times after flare screening. For the PN instrument, only 6 pointings were used.

\subsection{HESS data}

In the HESS study (AH06), spectra were extracted from 14 square regions $\left(0.26^{\circ}\right.$ length, see Fig. 2$)$ covering the entire remnant. The data used in the spectral analysis have a cut on the minimum size of image of 80 photo-electrons resulting in a mean spatial resolution of $0.12^{\circ}$ (68\% containment radius). For the morphological analysis (radial profiles), the cut used in HESS 


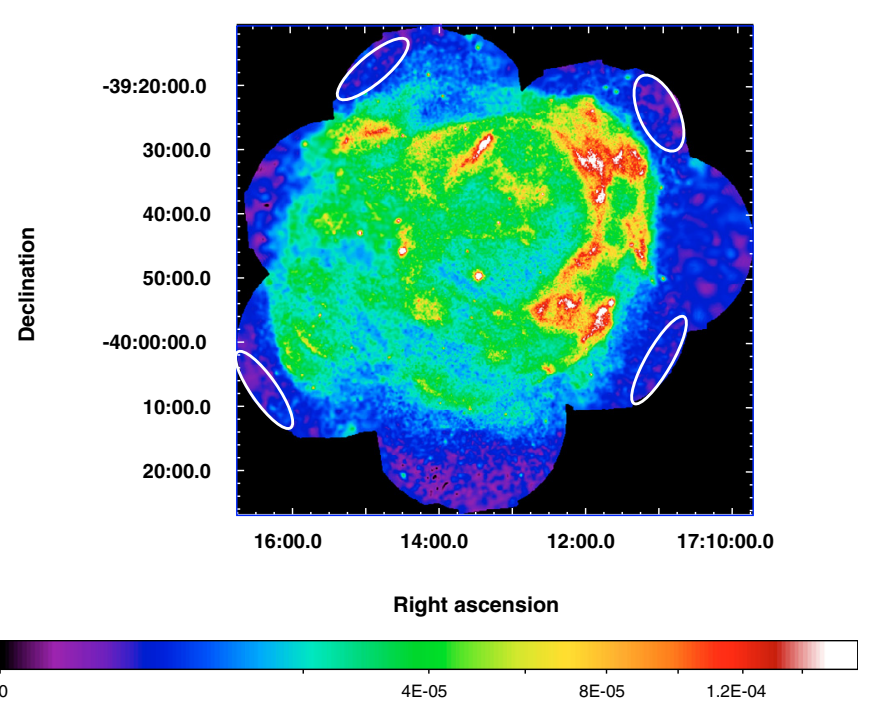

Fig. 1. EPIC MOS plus PN image in the $0.5-4.5 \mathrm{keV}$ band. The units are $\mathrm{ph} / \mathrm{cm}^{2} / \mathrm{s} / \operatorname{arcmin}^{2}$ and the scale is square root. The image was adaptively smoothed to a signal-to-noise ratio of 10 . The four ellipses show the regions used to estimate the local astrophysical background for the spectral analysis.

data was 200 photo-electrons which provided a higher spatial resolution of $0.08^{\circ}$. The detection efficiency varies over the $5^{\circ}$ of the full field of view but is almost constant at the scale of the remnant, which is $\sim 1^{\circ}$ wide (it varies by only $5 \%$ between the center and the edge of the object). All the data used in our study were taken from AH06 and we did not reprocess any of the $\mathrm{TeV}$ data. To compare the spectral properties, we used the $\gamma$-ray results presented in Table 2 of AHO6 and to compare the radial profiles, we used the data presented in Fig. 16.

\section{X-ray processing}

\subsection{Mosaic construction}

The mosaic was compiled in counts and an adaptive smoothing was applied so that the signal-to-noise ratio is at least 10. The instrumental background was derived from a compilation of blank sky observations (Carter \& Read 2007), renormalized in the 10-12 keV energy band for the MOS cameras (12-14 keV for $\mathrm{PN}$ ) and subtracted from each image. To derive the resulting image in units of photons $/ \mathrm{cm}^{2} / \mathrm{s} / \mathrm{pixel}$ instead of counts/s/pixel, the exposure map of each observation was multiplied by the average effective area in the energy band (assuming the same spectrum over the field of view). A mosaic of those exposure maps was then created and smoothed in the same way. The final image is the division of the counts mosaic by the exposure map mosaic.

The resulting image, which is the sum of the MOS and available PN data after flare screening, is presented in Fig. 1. The morphology of RX J1713.7-3946 can be decomposed in two main kinds of structures: diffuse emission present over all the remnant and bright filaments particularly visible in the west and north of the remnant (see Fig. 1). With the high sensitivity of $X M M-N e w t o n$, we can clearly see the faint emission in the observations of the regions south, east, and north. In particular, in the northern region of the remnant we can distinctly see a straight edge that is neither an artifact due to a CCD gap or any instrumental effect nor is it due to X-ray absorption along the line of sight because this straight edge remains visible in the 4.5-7.5 keV image (in this energy band the absorption is weak).
Table 1. XMM-Newton observations used in this paper.

\begin{tabular}{llccc}
\hline \hline & & \multicolumn{3}{c}{ Exposure (ks) } \\
& & MOS & PN \\
ObsId & Observation date & Total & Good & Good \\
\hline 0093670101 (NE) & 2001 September 5 & 15.3 & 1.8 & 0 \\
0093670201 (NW) & 2001 September 5 & 15.3 & 6.7 & 0 \\
0093670301 (SW) & 2001 September 8 & 15.3 & 15.2 & 10.0 \\
0093670401 (SE) & 2002 March 14 & 14.1 & 11.6 & 5.1 \\
0093670501 (CE) & 2001 March 2 & 13.8 & 13.0 & 6.5 \\
0207300201 (CE) & 2004 February 22 & 31.5 & 12.4 & 0. \\
0203470401 (NE) & 2004 March 25 & 17.0 & 16.1 & 6.7 \\
0203470501 (NW) & 2004 March 25 & 18.0 & 13.1 & 9.7 \\
0502080101 (E) & 2007 September 15 & 34.6 & 5.8 & 0 \\
0502080301 (W) & 2007 October 3 & 8.9 & 2.8 & 0 \\
0551030101 (S) & 2008 September 27 & 24.9 & 24.5 & 20.8 \\
\hline
\end{tabular}

The total and good columns represent the exposure time before and after flare screening.

The emission simply seems fainter there. Above that edge, we see a structure (in blue-green) that seems to represent the continuity of the shock.

The estimate of the astrophysical background is not simple in RX J1713.7-3946 as it seems to vary around the remnant. However to approximate the background level, we extracted the flux outside a circle of $0.56^{\circ}$ radius centered on the remnant $\left(\alpha_{\mathrm{J} 2000}=17 \mathrm{~h} 13 \mathrm{~m} 46 \mathrm{~s}, \delta_{\mathrm{J} 2000}=-39^{\circ} 44^{\prime} 56^{\prime \prime}\right)$. We then subtracted the mean value of this flux $\left(4.5 \times 10^{-6}\right.$ photons $\left./ \mathrm{cm}^{2} / \mathrm{s} / \mathrm{arcmin}^{2}\right)$ for the morphological study. The small structures of the SNR are not affected by this background subtraction.

\subsection{Spectral extraction method}

Because of the high spatial resolution, the XMM-Newton telescope can carry out spectral study on small scale, whereas in $\gamma$-rays the spectral analyses are completed on larger scale because of the comparatively lower spatial resolution of the HESS telescopes. To address this problem, we took into account the different point spread functions (PSFs) and the variation in the detection efficiency across the field of view of the two instruments.

In the case of XMM-Newton, the detection efficiency of the MOS and PN cameras can drop by $35 \%$ from the center to the edge of our $0.26^{\circ}$ extraction regions. For the HESS telescopes, the detection efficiency is almost constant for this region size (see Sect. 2.4). When extracting an X-ray spectrum in this large region, we want all the events to contribute with the same weight to the spectrum. To address this problem, we used the weight method described in Arnaud et al. (2001), where each event was corrected for its efficiency loss as a function of its position on the camera and its energy.

We also have to take into account the different size of the PSF of both instruments. For the spectral study of this SNR, the mean spatial resolution of the HESS instrument is $0.12^{\circ}(68 \%$ containment radius), which is comparable to the size of the extraction region $\left(0.26^{\circ}\right)$ whereas for the XMM-Newton observatory, the spatial resolution is about 7 arcsecs.

We assumed that the PSF of the HESS telescopes is a Gaussian of $\sigma=0.0795^{\circ}$ (corresponding to the HESS $68 \%$ containment radius of $0.12^{\circ}$ ) and that in comparison the PSF of XMM-Newton is negligible. To decrease the spatial resolution of the X-ray data, we redistributed randomly the position of each X-ray event according to the Gaussian probability density function. 

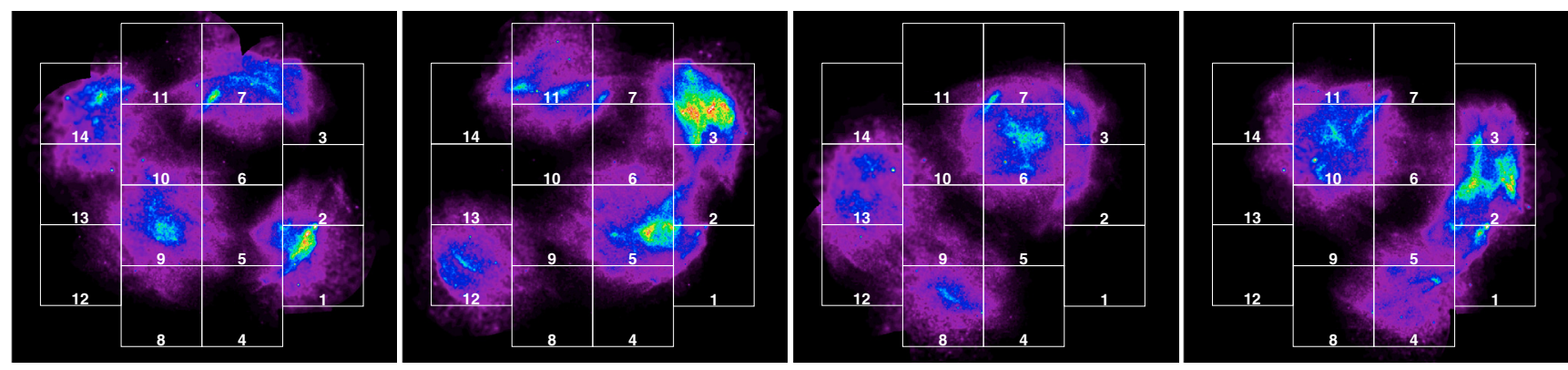

$2 \mathrm{E}-05$

$4 \mathrm{E}-05$

$6 \mathrm{E}-05$

$8 \mathrm{E}-05$

$1.2 \mathrm{E}-04$

Fig. 2. Spatial X-ray contribution to each HESS region (as defined in Aharonian et al. 2006). We see that due to the size of the point spread function comparable with the size of the extraction regions, many events outside the region contribute to the spectrum. To save space, each frame shows the contributions to three or four separate regions at once. The linear scale is in $\mathrm{ph} / \mathrm{cm}^{2} / \mathrm{s} / \mathrm{arcmin}^{2}$. The regions 1 to 14 defined here are used later for the spectral analysis in Table 2, Fig. 7-9.

For the purpose of spectral comparison with the $\gamma$-rays, we removed the two following very bright point-like sources in the X-ray data: 1WGA J1713.4-3949, which is argued to be the Central Compact Object of the SNR (Slane et al. 1999; Lazendic et al. 2003; Cassam-Chenaï et al. 2004), and 1WGA J1714.4-3945, which is associated with a star (Pfeffermann \& Aschenbach 1996).

\subsection{X-ray background spectra}

The instrumental background spectrum is derived from blank sky observations (Carter \& Read 2007) in the same detector area, renormalized in the 10-12 keV energy band for the MOS cameras (12-14 keV for PN) and processed with the same method as the observations (see Sect. 3.2).

The subtraction of the local astrophysical background, is not a simple problem in RX J1713.7-3946. On the one hand, since the remnant is $\sim 1^{\circ}$ in size and close to the Galactic plane, the local astrophysical emission can vary around the remnant. On the other hand, there are only a few pointings that allow us to estimate this background. We extracted background spectra outside the SNR in 4 regions where the statistics were sufficient (see the 4 ellipses in Fig. 1). In this astrophysical background, no emission lines are seen but the statistics is low. To compare the spectral properties of the background between the different regions, we modeled the spectra using a simple absorbed power law model. The parameters of this model are similar in the northern, north-western, and south-western regions, i.e. $N_{\mathrm{H}} \simeq 0.4 \times 10^{22} \mathrm{~cm}^{-2}$, index $\simeq 1.9$ and a normalization norm $\simeq 1.4 \times 10^{-3} \mathrm{keV}^{-1} \mathrm{~cm}^{-2} \mathrm{~s}^{-1}$ at $1 \mathrm{keV}$ (renormalized to the area of the large HESS extraction regions). We note that in the south-eastern region, the normalization parameter is $40 \%$ lower. Since all the extracted background spectra have low statistics, we decided to use a power law model with the parameters listed above for the astrophysical background instead of a spectrum. We used the same $N_{\mathrm{H}}$, index and normalization parameters for the northern, north-western, and south-western regions for all the extraction regions. The impact of this choice for the southeastern region, where the normalization is lower, is discussed later in Sect. 3.4.

\subsection{Spectral modeling}

The X-ray emission of the remnant is dominated by a nonthermal continuum and no emission lines have been detected so
Table 2. Best-fit X-ray parameters obtained with an absorbed power law model for the HESS regions defined in Fig. 2.

\begin{tabular}{cccc}
\hline \hline Region & $\begin{array}{c}N_{\mathrm{H}} \\
10^{22} \mathrm{~cm}^{-2}\end{array}$ & Index & $\begin{array}{c}\text { Integrated flux } \\
\left(10^{-2} \mathrm{~cm}^{-2} \mathrm{~s}^{-1}\right)\end{array}$ \\
\hline 1 & 0.79 & $2.43(2.42-2.44)$ & $0.91(0.90-0.92)$ \\
2 & 0.62 & $2.35(2.34-2.35)$ & $1.58(1.57-1.59)$ \\
3 & 0.72 & $2.35(2.35-2.36)$ & $1.74(1.73-1.75)$ \\
4 & 0.76 & $2.37(2.37-2.39)$ & $0.76(0.75-0.77)$ \\
5 & 0.59 & $2.32(2.31-2.32)$ & $1.42(1.41-1.43)$ \\
6 & 0.56 & $2.44(2.43-2.44)$ & $1.44(1.43-1.45)$ \\
7 & 0.67 & $2.41(2.40-2.42)$ & $0.89(0.87-0.90)$ \\
8 & 0.64 & $2.23(2.22-2.24)$ & $0.58(0.57-0.58)$ \\
9 & 0.48 & $2.22(2.22-2.23)$ & $0.90(0.89-0.91)$ \\
10 & 0.46 & $2.32(2.31-2.33)$ & $1.15(1.14-1.16)$ \\
11 & 0.63 & $2.35(2.34-2.36)$ & $0.72(0.72-0.74)$ \\
12 & 0.50 & $2.17(2.15-2.18)$ & $0.51(0.50-0.52)$ \\
13 & 0.51 & $2.31(2.30-2.32)$ & $0.69(0.68-0.70)$ \\
14 & 0.57 & $2.28(2.26-2.29)$ & $0.70(0.68-0.70)$ \\
\hline
\end{tabular}

${ }^{a}$ Corrected for absorption.

The X-ray flux is integrated in the $1-10 \mathrm{keV}$ band.

far (Koyama et al. 1997; Slane et al. 1999; Pannuti et al. 2003; Cassam-Chenaï et al. 2004). In our study, all spectra are well described by an absorbed power law model as illustrated in Fig. 3. In some observations, the PN instrument exposure time was null after flare screening. To ensure homogeneous coverage of the remnant, we retained only the MOS data. Whenever it was possible, we fitted independently, as a test, the spectrum extracted from the MOS1\&2 and PN instruments and compared the resulting best-fit model parameters. They agreed within the statistical errors bars.

All the data were fitted using unbinned spectra with the C-statistic implemented in Xspec (v12.3.1). Binning was used for graphical purposes only and fixed at $3 \sigma$ for all spectra. We fitted all the data from $0.8 \mathrm{keV}$ to $10 \mathrm{keV}$. The best-fit model parameters for the HESS regions are listed in Table 2. As discussed in Sect. 3.3, the local astrophysical background in the southeastern region seems weaker than the average background used for all the regions. Therefore, we studied the impact of those different backgrounds for the faintest region (the most sensitive to background subtraction), which is located in the SE, i.e. region 12 . When using a value of the normalization parameter that is $40 \%$ lower for the background model, the new best-fit model parameters do not vary much. While the hydrogen column and the index change within the errors bars, the integrated X-ray flux 


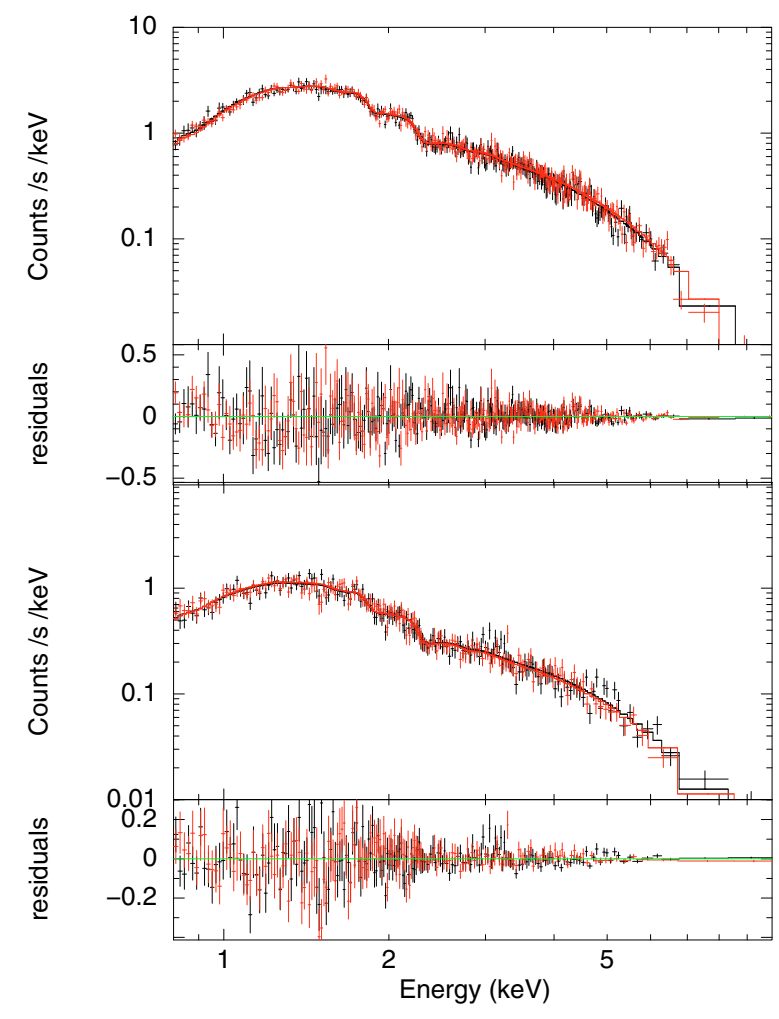

Fig. 3. Best-fit model X-ray spectrum from region 3 (Top panel) and region 12 (Bottom panel) with an absorbed power law model (MOS1 spectrum is in black and MOS2 in red). The region 3, located at the north-west, is the brightest region of the remnant. It has a steep spectrum (2.35) and high absorption $\left(0.72 \times 10^{22} \mathrm{~cm}^{-2}\right)$. On the south-east of the remnant, region 12 is faint, has a weak absorption $\left(0.50 \times 10^{22} \mathrm{~cm}^{-2}\right)$ and has a harder spectrum (2.17).

varies by less than $10 \%$. Since the variations are small and do not affect the results of our study, we kept the same astrophysical background for all the regions.

\subsection{Radial profile method}

Since we now have a full coverage of the remnant, we can accurately compare the radial profiles of the emission of the remnant in X-and $\gamma$-rays. To match the HESS spatial resolution, we smoothed the X-ray image with a Gaussian of $\sigma=0.053^{\circ}$ (corresponding to $r_{68 \%}=0.08^{\circ}$ ). We then extracted a flux per unit solid angle as a function of the distance from the center of the smoothed X-ray images (1-2 keV and 2-4.5 keV band) in eight sectors as shown in Fig. 4. To compare the radial profiles at both wavelengths, the X-ray profiles were scaled by a unique normalisation factor calculated to be the ratio of the total number of counts in $\gamma$-rays to the total flux in X-rays for the entire remnant. In the last two bins of some X-ray profiles, there is not enough coverage to estimate the flux. These bins are removed from the profile. As in the spectral study, the two brightest pointlike sources (see Sect. 3.2) were removed from the X-ray images. The resulting radial profiles are shown in Fig. 10 and discussed in Sect. 4.3.2.

\section{Results}

\subsection{Radio flux}

An accurate estimate of the integrated radio flux density is critical for investigating the mechanisms responsible for the

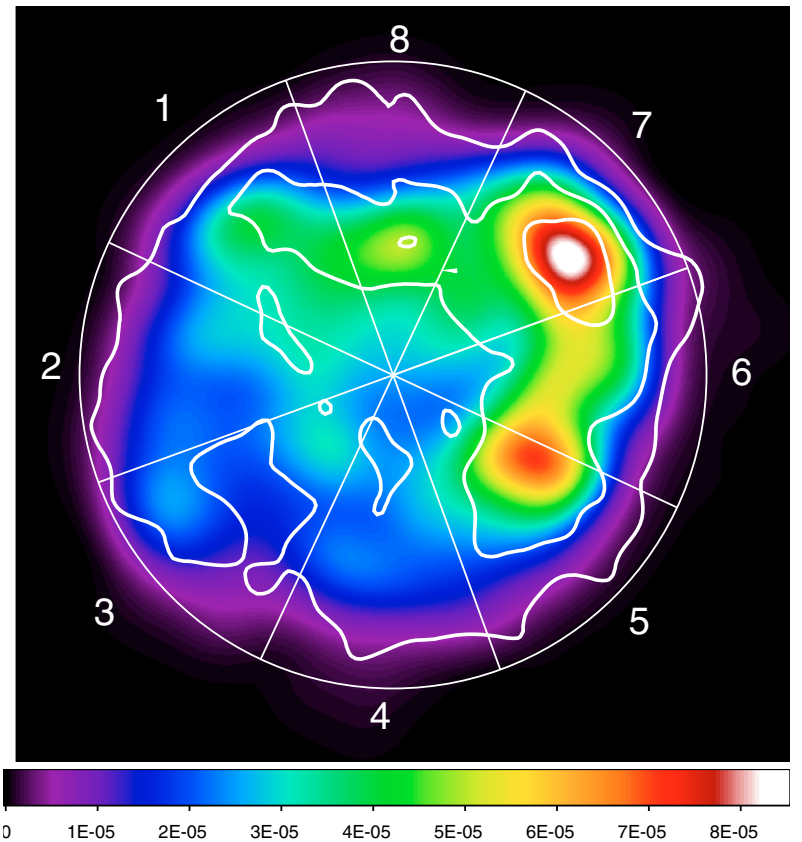

Fig. 4. Same X-ray mosaic as Fig. 1 smoothed to match the point spread function of the HESS telescopes. The scale is linear and units are in $\mathrm{ph} / \mathrm{cm}^{2} / \mathrm{s} / \mathrm{arcmin}^{2}$. Overlaid are the $\gamma$-ray contour excess (spaced at 30, 60, and 90 counts) from Fig. 7 of Aharonian et al. (2006). The sectors used for the radial profile comparison of Fig. 10 are also drawn.

high-energy emission. Following two different paths, we reanalyze the available radio data at $1.4 \mathrm{GHz}$ to estimate the radio flux density of RX J1713.7-3946.

Since the largest well imaged structure for the ATCA observations is 25 arcmin in size, to recover information about structures larger than this size, we must combinate these data with single dish observations. However, in this case, it was impossible to complete the procedure because the overlap annulus of the interferometric and single antenna data set ${ }^{1}$ in the uv space is too small to produce a reliable image. We therefore used the single dish data at $1.4 \mathrm{GHz}$ to estimate the integrated flux density within the area covered by the X-ray emission associated with the SNR (outer contour depicted in Fig. 5). This estimate provides an upper limit to the flux density of about $26 \mathrm{Jy}$ with an uncertainty of the order of $10 \%$. In addition, we integrated over an interferometric image based on ATCA data within the same outer contour. In this case, since the largest well imaged structure at this frequency is about 25 arcmin, we corrected "by hand" by adding the minimum flux density required to fill in the few negatives remaining in the image. The surface brightness corresponding to this addition was below $7.4 \times 10^{-24} \mathrm{~W} \mathrm{~m}^{-2} \mathrm{~Hz}-1 \mathrm{sr}^{-1}$. The off-source rms-noise is about $0.7 \mathrm{mJy} /$ beam and the constant value added to fill in the few negatives is $1.2 \mathrm{mJy} / \mathrm{beam}$. This correction amounts to less than $1 \%$ of the total estimated flux density.

Whether the radio emission from Arc 2 (see Fig. 5) is thermal or non-thermal in nature is an important issue because it is one of the brightest features within the X-ray contours. In order to investigate the connection of Arc 2 with the SNR, we used mid-infrared observations. The right-hand panel of Fig. 5

\footnotetext{
1 The only public single dish data available at this frequency come from the survey of the south celestial hemisphere carried out with the $30 \mathrm{~m}$ dish of the Argentine Institute of Radio Astronomy (IAR Testori et al. 2001) (HPBW $~ 34$ arcmin at $1.4 \mathrm{GHz}$ ).
} 

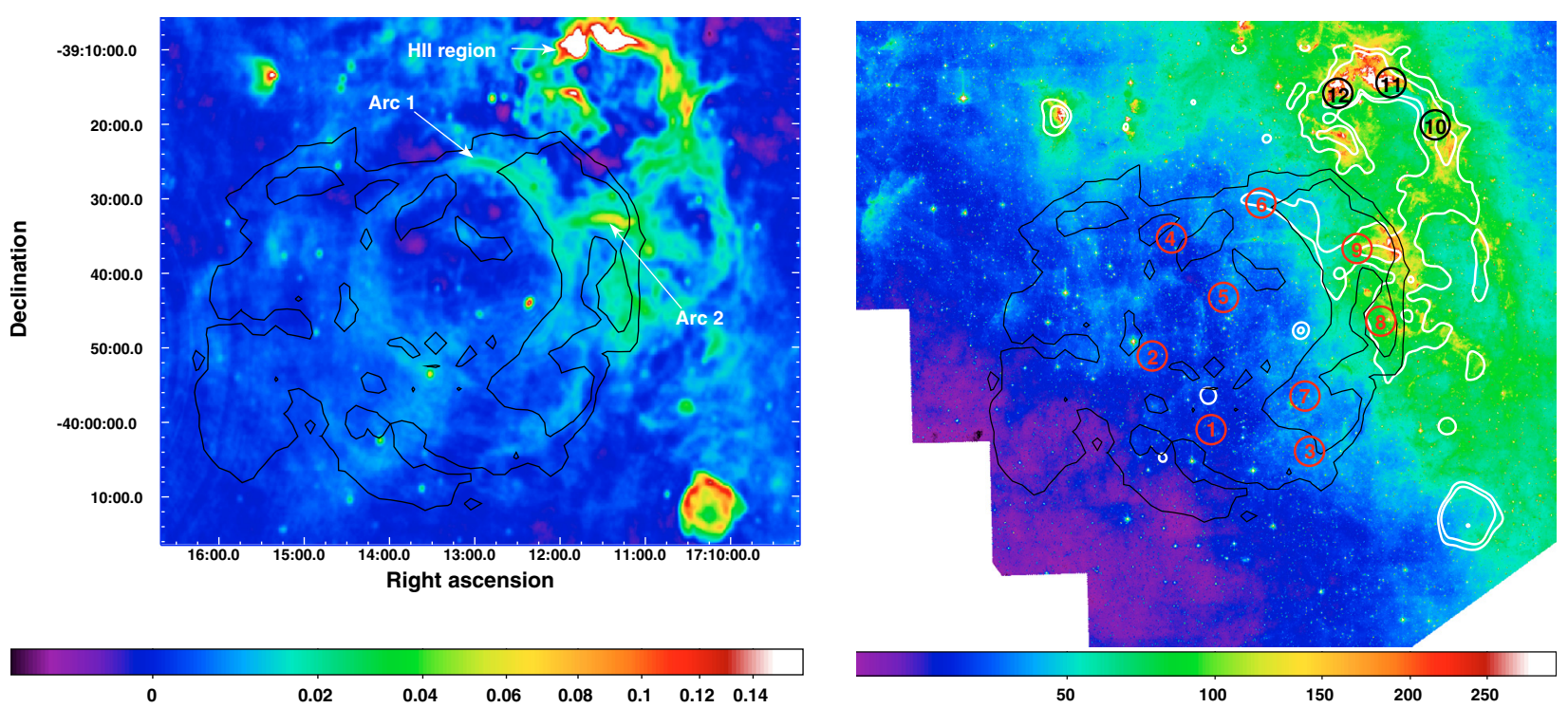

Fig. 5. Left: radio image of the region of RX J1713.7-3946 at $1.4 \mathrm{GHz}$ (from Lazendic et al. 2004). The scale is square root and units are in $\mathrm{Jy} \mathrm{beam}^{-1}$. Right: Spitzer image at $8 \mu \mathrm{m}$ from the GLIMPSE survey. The X-ray contours are represented in black in both images and the radio contours in white. The regions used for the color-color plot of Fig. 6 are labeled here. The scale is square root and units are in $\mathrm{MJy} \mathrm{sr}^{-1}$.

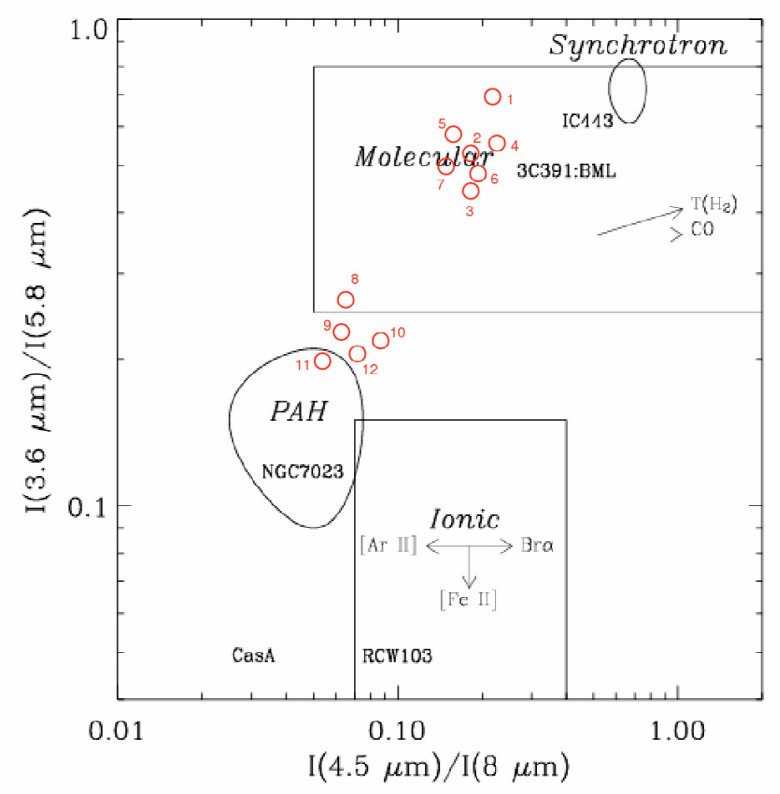

Fig. 6. Infrared color-color diagram for the regions defined in Fig. 5 (Right) overlaid on Fig. 2 of Reach et al. (2006). Two distincts groups can be seen. The emission from the regions 8 to 12 is compatible with PAH origin including our region of interest Arc 2 (labeled as region 9 here). Therefore, the radio emission from Arc 2 is likely to be thermal and unrelated to the remnant.

shows Spitzer $8 \mu \mathrm{m}$ mid-infrared emission in the direction of RX J1713.7-3946. Particular intense infrared emission is visible at the location of the HII region G347.611+0.204. The infrared emission also provides evidence of a good morphological correlation with the radio Arc 2 suggesting a thermal origin for this feature. To investigate the nature of Arc 2 we applied the color-color criteria proposed by Reach et al. (2006) based on Spitzer data obtained at 3.6, 4.5, 5.8, and $8 \mu \mathrm{m}$, finding that Arc 2 has color characteristics compatible with those of polycyclic

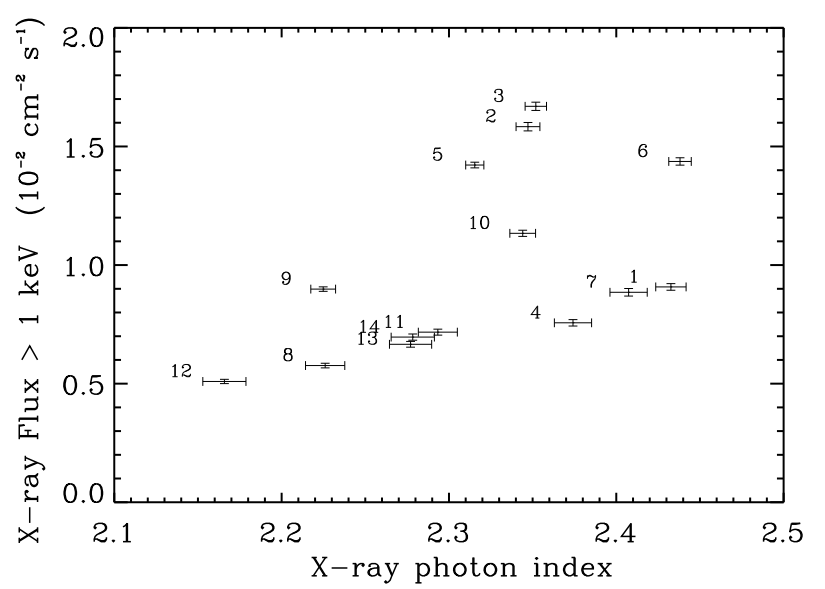

Fig. 7. Correlation plot between the X-ray photon index and the X-ray integrated flux (in the $1-10 \mathrm{keV}$ band). The parameters shown here are the average of MOS1 and MOS2 best-fit model parameters. The label over each point corresponds to HESS regions as defined in Fig. 2.

aromatic hydrocarbons (PAHs) origin (see Fig. 6). We applied $\mathrm{HI}$ absorption techniques based on data extracted from the southern galactic plane survey (SGPS; McClure-Griffiths et al. 2005) together with a flat rotation model for our Galaxy (assuming as solar parameters $R_{\odot}=7.6 \pm 0.3 \mathrm{kpc}$ and $\Theta_{\odot}=214 \pm 7 \mathrm{~km} \mathrm{~s}^{-1}$ ). From this study, we conclude that the most distant HI absorption feature for the Arc 2 is at $-120 \mathrm{~km} \mathrm{~s}^{-1}$, which corresponds to a near distance of $\sim 6.7 \mathrm{kpc}$, placing in principle this thermal arc beyond RX J1713.7-3946. With this information, we estimated the associated radio flux density by subtracting the contribution from all overlapping radio point sources (likely to be extragalactic and/or compact HII regions) and from Arc 2 (about 1.5 Jy) because it is likely to be unrelated to the remnant, estimating a lower limit to the total flux density of $\sim 22 \mathrm{Jy}$. Therefore, we conclude that the associated radio flux density at $1.4 \mathrm{GHz}$ is between 22 and $26 \mathrm{Jy}$. 


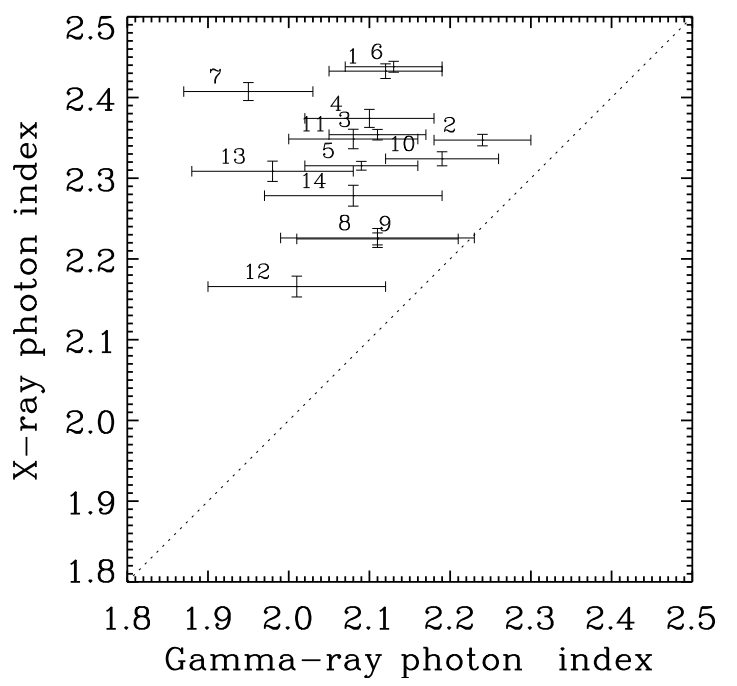

Fig. 8. X-ray photon index against $\gamma$-ray photon index. A systematic error of 0.1 is to be added to the $\gamma$-ray photon index. The dashed line is the bisector.

\subsection{Global $X$-ray flux}

We extracted a MOS spectrum from the entire remnant without degrading the spatial resolution of the data. The best-fit parameters (with background subtraction as described in Sect. 3.3) are $N_{\mathrm{H}}=0.66 \pm 0.01 \times 10^{22} \mathrm{~cm}^{-2}$, index $=2.37 \pm 0.01$, an absorbed 1-10 keV flux of $3.95 \pm 0.03 \times 10^{-10} \mathrm{erg} \mathrm{cm}^{-2} \mathrm{~s}^{-1}$, and a non-absorbed flux in the same band of $5.23 \pm 0.04 \times$ $10^{-10} \mathrm{erg} \mathrm{cm}^{-2} \mathrm{~s}^{-1}$. Our main source of uncertainty is the absolute calibration of XMM-Newton, which is known to a precision of $10 \%$ rather than the very low statistical error (less than $1 \%$ ). Another flux estimate is obtained with a srcut synchrotron model using the radio flux upper limit derived in Sect. 4.1. The nonabsorbed flux derived is $5.49 \pm 0.05 \times 10^{-10} \mathrm{erg} \mathrm{cm}^{-2} \mathrm{~s}^{-1}$, very similar to that obtained with a power law model. In the srcut model, the radio spectral index was fixed to be 0.6 and a break frequency of $4.2 \times 10^{17} \mathrm{~Hz}$ was derived. This value, averaged across the remnant, is similar to the maximum break frequency reached in the bright limbs of SN 1006 (Rothenflug et al. 2004).

We note that the 1-10 keV non-absorbed flux obtained by Tanaka et al. (2008) with Suzaku is $47 \%$ higher $(7.65 \times$ $10^{-10} \mathrm{erg} \mathrm{cm}^{-2} \mathrm{~s}^{-1}$ ) than what we have found. However the absorption and index of their spectrum $\left(0.79 \times 10^{22} \mathrm{~cm}^{-2}\right.$ and 2.39 respectively) agree with our parameters. We note that whereas our spectrum is extracted directly on the entire remnant, the Suzaku spectrum is the sum of spectra from 10 particular regions scaled up to the entire remnant assuming the surface brightness from the ASCA image (Sect. 3.3 of Tanaka et al. 2008). In our spectrum, the point sources were removed but their contribution to the total flux is weak (less than $1 \%$ for the central compact object). We cross-checked the value of our absorbed global flux derived from the spectrum (in the $0.5-4.5 \mathrm{keV}$ energy band) to that derived from our mosaiced image. Both fluxes agree within $5 \%$.

\section{3. $X$ - and $\gamma$-ray comparison}

\subsubsection{Spectral results}

The best-fit model parameters of the X-ray spectral modeling of the 14 regions are given in Table 2 . The large variation in photon index $(1.9<\Gamma<2.6)$ seen in X-rays when using small extraction regions in Cassam-Chenaï et al. (2004) were reduced

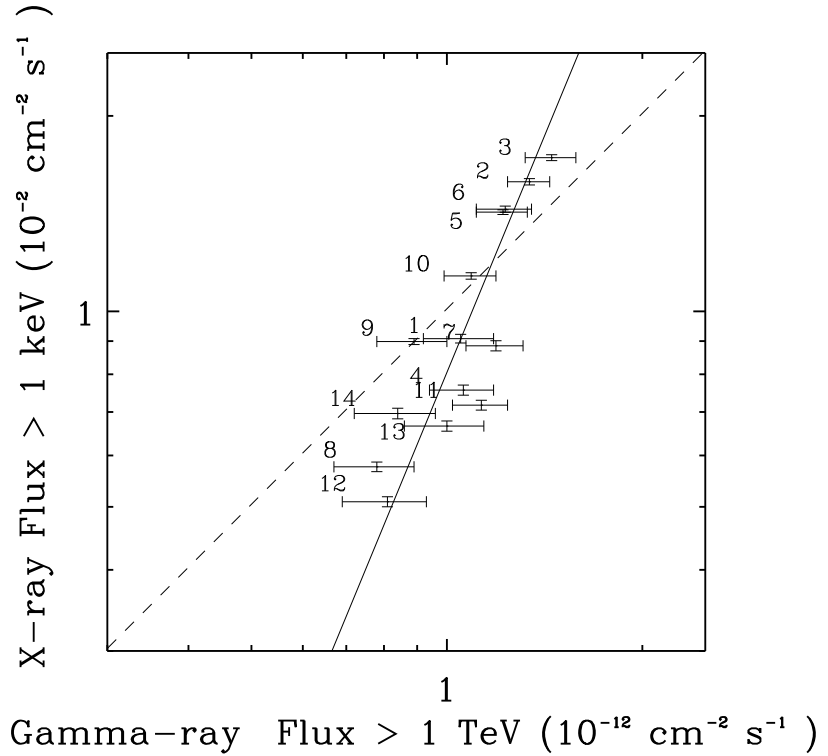

Fig. 9. Log-log correlation plot between the HESS $\gamma$-ray integrated flux (1-10 TeV band) and the XMM-Newton X-ray integrated flux (1-10 keV band, using MOS $1 \& 2$ data). We can see that the correlation is best-fitted with a non-linear function $F_{v}^{\mathrm{X}}=0.81 \pm 0.09\left(F_{v}^{\gamma}\right)^{2.41 \pm 0.55}$ (solid line). The best-fit linear function $F_{v}^{\mathrm{X}}=1.01 \pm 0.04 F_{v}^{\gamma}$ is also represented (dashed line).

significantly by using larger extraction regions and a degraded spatial resolution for the X-ray data $(2.2<\Gamma<2.4$, see Fig. 7$)$. The comparison of the $\mathrm{X}$ - and $\gamma$-ray photon index (Fig. 8) shows no significant correlation. The distribution of the photon index in X- and $\gamma$-rays has a mean value of 2.32 and 2.09 , respectively, and a standard deviation of 0.075 and 0.073 respectively. Whereas the dispersion of the photon index at both energies is the same, the X-ray index is slightly higher than the $\gamma$-ray one. However, there is a systematic error in the $\gamma$-ray photon index of 0.1 (AH06) that should be added to Fig. 8. Whereas the variations of the photon index are small, there are significant variations in the flux from the 14 large regions. Figure 9 shows a good correlation between the X-ray integrated flux (1-10 keV band) and the $\gamma$-ray integrated flux $(1-10 \mathrm{TeV}$ band). We note that the bright regions are brighter in X-rays than in $\gamma$-rays. This type of behavior has also been observed by Tanaka et al. (2008) with Suzaku. However, whereas they interpreted this as a linear correlation with some X-ray deviation, we interpret this as a non-linear correlation. We measured this non-linear correlation by placing the X-ray flux along the $\mathrm{X}$-axis (since the error bars in the X-ray flux are lower than that in $\gamma$-rays). This measured slope is then inverted to obtain $\mathrm{d} \log F_{v}^{\mathrm{X}} / \mathrm{d} \log F_{v}^{\gamma}$. The best-fit power law function $F_{\gamma}^{\mathrm{X}}=0.81 \pm 0.09\left(F_{\gamma}^{\gamma}\right)^{2.41 \pm 0.55}$ is statistically preferred $\left(\chi^{2} /\right.$ d.o.f. $\left.=9.28 / 12\right)$ over the best-fit linear function $F_{v}^{\mathrm{X}}=1.01 \pm 0.04 F_{v}^{\gamma}\left(\chi^{2} /\right.$ d.o.f. $\left.=61.06 / 13\right)$. We note that the fit with a linear function $F_{v}^{\mathrm{X}}=a F_{v}^{\gamma}-b$ (best-fit model parameters: $a=2.26$ and $b=1.43$ ) provides similar results in terms of $\chi^{2}\left(\chi^{2} /\right.$ d.o.f. $\left.=9.38 / 12\right)$ than the non-linear fit but is not physically understandable because both images are already background subtracted.

\subsubsection{Morphological results}

The comparison of the radial profiles allows us to investigate the extent of each type of emission as well as to localize their 


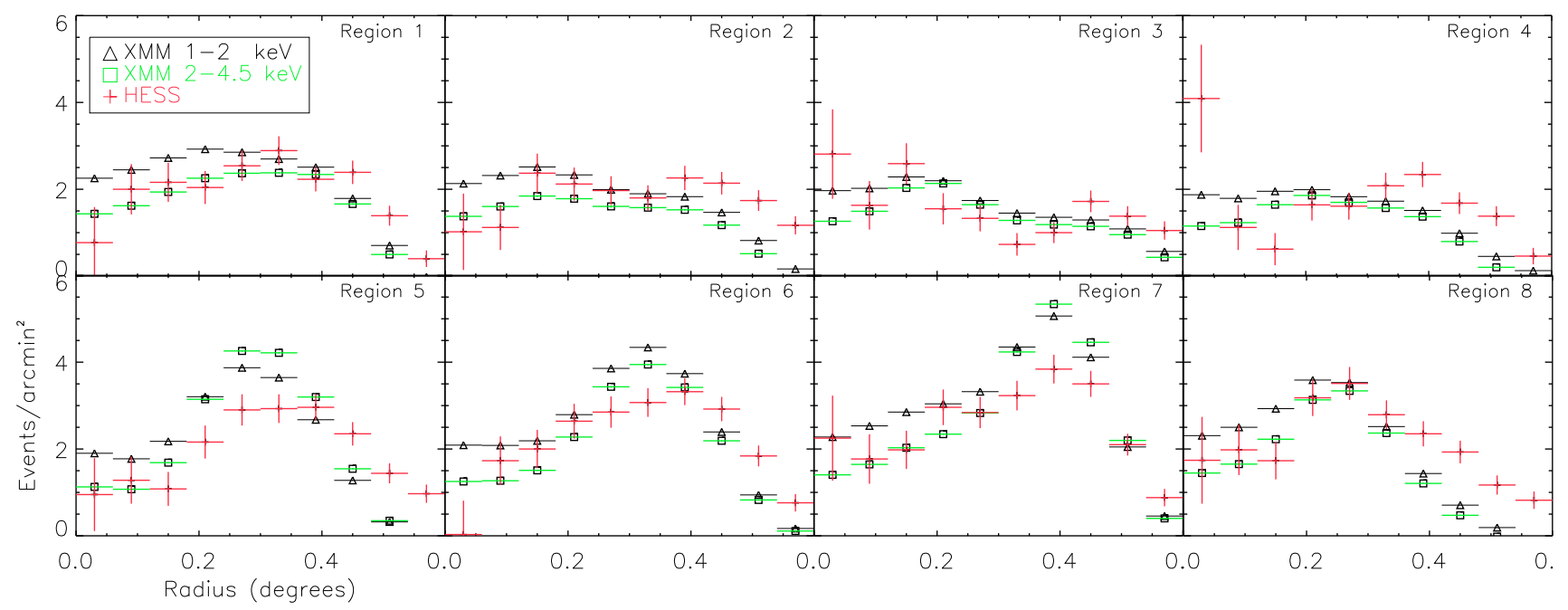

Fig. 10. Radial profiles in X-rays in two energy bands and in $\gamma$-rays for the 8 sectors defined in Fig. 4. The general agreement is good and particularly striking in sector 7 (the brightest spot of the remnant in both wavelengths). However, there are also interesting differences in sectors 5 and 6, where the bulk of the X-ray emission seems to come more from the inside of the SNR than in $\gamma$-rays.

respective peaks. This study has already been carried out by AH06 by comparing the ASCA and HESS data. However, since the coverage with ASCA did not always reach the boundaries of the SNR, it was impossible to compare accurately the extension of the remnant in both wavelengths.

The global agreement between the 8 sectors is good and particularly striking in sector 7 (the brightest spot of the remnant in both wavelengths) as can be seen in Fig. 10. In regions 5 and 6 , we see that the peak in the emission, along the radial direction, is located closer to the center in X-rays than in $\gamma$-rays. The global shape of the radial profile is similar at both wavelengths but shifted by $\sim 0.1^{\circ}$ to the center in X-rays.

It is important to note that we subtracted an astrophysical background to the X-ray radial profiles. To estimate the background, we used the same region as in the mosaic building section (Sect. 3.1).

\section{Discussion}

\subsection{Global synchrotron spectrum}

The radio flux density at $1.4 \mathrm{GHz}$ that we derive in our study (22 Jy $<S<26 \mathrm{Jy}$ ) for the entire remnant is significantly higher than the flux density previously published. However, it is important to note that the flux calculated by Ellison et al. (2001) ( $S=4 \pm 1 \mathrm{Jy}$ ) was estimated only locally for the two bright arcs in the northwestern region. Later on, Lazendic et al. (2004) derived for these features a flux density of $6.7 \pm 2.0 \mathrm{Jy}$ based on the improved radio image at $1.4 \mathrm{GHz}$. In X-rays, the contribution of the northwestern region accounts for $\sim 20 \%$ of the total flux in the $0.5-4.5 \mathrm{keV}$ energy band. If the ratio of synchrotron emission in X-rays to that in radio is more or less constant throughout the remnant, the radio flux expected for the entire remnant is of the order of $26 \mathrm{Jy}^{2}$, which is compatible with our estimation. We note that the flux that we derive is about 2 times higher than the flux of 13.4 Jy used in AH06. This flux density was obtained by assuming that the flux density for the entire remnant was about

\footnotetext{
2 Without taking into account the thermal emission from Arc 2 that accounts for about $1.5 \mathrm{Jy}$ out of the $6.7 \mathrm{Jy}$ estimation from Lazendic et al. (2004).
}

twice the flux density value obtained by Lazendic et al. (2004) for the northwestern region. Our higher new estimate tends to reduce the difference between the measurements and predictions of the hadronic models usually requiring high magnetic fields $>100 \mu \mathrm{G}$ (Tanaka et al. 2008).

In X-rays, the 1-10 keV non-absorbed flux that we derive with XMM-Newton is significantly lower than the value derived by Tanaka et al. (2008) with Suzaku (5.23 and $7.65 \times$ $10^{-10} \mathrm{erg} \mathrm{cm}^{-2} \mathrm{~s}^{-1}$ respectively, see Sect. 4.2). This new X-ray flux and the higher radio flux estimate decrease the global X-ray to radio ratio by about a factor of 3 . This change is clearly important to the shape of the synchrotron spectral energy distribution and could impact the results from multiwavelength models of the remnant.

\section{2. $X$ - and $\gamma$-ray comparison}

\subsubsection{Spectral index}

When using large extraction regions and degrading the spatial resolution of the X-ray data, we found no significant spatial variation in the photon index (see Sect. 4.3.1). In other words, the small-scale variations reported by Cassam-Chenaï et al. (2004) are not resolved at the resolution of the HESS telescope. If small-scale spectral variations in $\gamma$-rays exist, one can expect them to be washed out as well with the current HESS resolution. However the mean X-ray photon index (2.32) is slightly steeper than the $\gamma$-ray one (2.09). In the case of inverse Compton (IC) off the CMB photons for the $\gamma$-ray emission, the mean electron energy required to produce a photon at $1 \mathrm{TeV}$ is $E_{\mathrm{e}}^{\mathrm{IC}}=16 \mathrm{TeV}$ (for inverse Compton off IR or optical photons it is of course lower). The electrons emitting synchrotron radiation at $1 \mathrm{keV}$ with a magnetic field of $70 \mu \mathrm{G}$ have an energy of $E_{\mathrm{e}}^{\mathrm{sync}}=27 \mathrm{TeV}$. At those energies, the electrons are close to the cutoff and we expect a higher index when considering electrons of higher energies. We can reasonably set an upper limit to the magnetic field since $E_{\mathrm{e}}^{\mathrm{sync}}$ should not be lower than $E_{\mathrm{e}}^{\mathrm{IC}}$ to reproduce the steeper index of the synchrotron spectra. This sets an upper limit to the downstream magnetic field of $200 \mu \mathrm{G}$, which is consistent with the estimate based on the width of the X-ray filaments (about $70 \mu \mathrm{G}$ from Ballet 2006; Berezhko \& Völk 2006). 


\subsubsection{Correlation between the X-ray and $\gamma$-ray fluxes}

In Sect. 4.3.1, we found that the X-ray flux is indeed correlated with the $\gamma$-ray flux, but the correlation appears non-linear, more like $\mathrm{d} \log F_{v}^{\mathrm{X}} / \mathrm{d} \log F_{v}^{\gamma}=2.41 \pm 0.55$ (Fig. 9). In this section, we discuss why it should be so. We assume that most of the range in flux that we see is caused by to density variations around the remnant. It is certainly not entirely true (geometric effects such as limb brightening must play a role at some point) but it is probably indeed the major contributor in view of the very structured interstellar gas and X-ray image. We assume that for the range of energies corresponding to the emission of $\gamma$ and X-rays (i.e. between one and a few hundred $\mathrm{TeV}$ ), the particle distribution can be represented as a cut-off power law $\mathrm{d} N / \mathrm{d} E=K E^{-s} C\left(-E / E_{\mathrm{c}}\right)$ in which $K$ is proportional to the ambient density, and $E_{\mathrm{c}}$ is limited by synchrotron losses for electrons $\left(E_{\mathrm{c}, \mathrm{e}} \propto V_{\mathrm{sh}} / \sqrt{B_{\mathrm{d}}}\right)$ and by age for protons $\left(E_{\mathrm{c}, \mathrm{p}} \propto B_{\mathrm{d}} V_{\mathrm{sh}}^{2}\right)$, where $B_{\mathrm{d}}$ is the downstream magnetic field. The shape of the cutoff $\mathrm{C}(x)$ can be any function decreasing from 1 to 0 . We also assume that the magnetic field may increase with density $\left(B_{\mathrm{d}} \propto n^{\beta}\right)$ and that the shock velocity varies as $V_{\text {sh }} \propto n^{-0.5}$ (same pressure).

By calculating $\mathrm{d} \log F_{v} / \mathrm{d} \log n$ for the synchrotron and inverse Compton case (see Appendix A), we can then predict a flux-flux correlation comparable to that evident in Fig. 9:

$$
\frac{\mathrm{d} \log F_{v}^{\mathrm{sync}}}{\mathrm{d} \log F_{v}^{\mathrm{IC}}}=\frac{\frac{s+1}{2}(1+\beta)-\alpha_{\mathrm{X}}}{1-(1+\beta)\left(\alpha_{\gamma}-\frac{s-1}{2}\right)},
$$

where $\alpha_{\mathrm{X}}$ and $\alpha_{\gamma}$ are respectively the $\mathrm{X}$ and $\gamma$-ray spectral slope in energy not photons $\left(\alpha_{i}=-\mathrm{d} \log F_{v}^{i} / \mathrm{d} \log v\right)$.

For a standard value of $s=2$ and the observed values of $\alpha_{\mathrm{X}}=1.32$ and $\alpha_{\gamma}=1.09$, this gives $(0.18+1.5 \beta) /(0.41-$ $0.59 \beta)$. If $B_{\mathrm{d}}$ is insensitive to density $(\beta=0)$, the predicted correlation is the opposite of what is seen: the range in X-ray flux would be smaller than in $\gamma$-rays, because the negative feedback on $v_{\mathrm{c}}$ due to $V_{\mathrm{sh}}$ is stronger in X-rays, which are further in the cutoff part of the spectrum. However, a modest dependence of $B_{\mathrm{d}}$ on density like $\beta=0.1$ is enough to invert the trend because, in the loss-dominated regime, the $\gamma$-ray cutoff frequency decreases with $B_{\mathrm{d}}$, while the X-ray one is independent of $B_{\mathrm{d}}$. In other words, the slope of the $\log \left(F_{\mathrm{X}}\right)$ versus $\log \left(F_{\gamma}\right)$ correlation is very sensitive to $\beta$. To derive the observed value of 2.41 , we require that $\beta=0.28$.

Because of the oversimplified character of that approach, we do not claim that this is a measurement of $\mathrm{d} \log B_{\mathrm{d}} / \mathrm{d} \log n$ but we assert that it demonstrates that such a steep correlation is reasonable in a leptonic model. The specific model in which we have tried to push the calculation further (Appendix B) does not provide a consistent answer, but it is far from unique. One way of improving the measurements would be to use extraction areas in which the filling factors of the SNR are the same, such as the angular sectors in Fig. 4. This would leave in the flux variations only what is due to varying external conditions. It requires reanalyzing the HESS data, so it is left for future work.

If we return to the hadronic hypothesis, the same line of reasoning (see Appendix A) implies that:

$$
\frac{\mathrm{d} \log F_{v}^{\text {sync }}}{\mathrm{d} \log F_{v}^{\text {hadr }}}=\frac{\frac{s+1}{2}(1+\beta)-\alpha_{\mathrm{X}}}{2-(1-\beta)\left(\alpha_{\gamma}+1-s\right)} .
$$

For a standard value of $s=2$ and the observed values of $\alpha_{\mathrm{X}}=1.32$ and $\alpha_{\gamma}=1.09$, this gives $(0.18+1.5 \beta) /(1.91+0.09 \beta)$. It is clear that regardless of the value of $\beta<1$ ( $B_{\mathrm{d}}$ is unlikely to increase more rapidly than density) this quantity is always $<1$.
In other words, no magnetic field can make up for the natural $n^{2}$ character of the hadronic mechanism, which predicts a more rapid increase of $F_{v}^{\text {hadr }}$ with density. In our (over)simplified framework, the correlation we observe therefore does not favor a hadronic model.

There remains the possibility (Malkov et al. 2005) that the density increases steeply outwards (SNR hitting a shell) to the point where most of the $\gamma$-ray emission is produced outside the remnant (in the precursor). In that case, the width of the precursor would increases with $E$ so that the spectral shape of the $\gamma$-ray emission may be estimated by multiplying $F_{v}^{\text {hadr }}$ by $E$ (Zirakashvili \& Aharonian 2007). This would alter Eq. (2) to

$\frac{\mathrm{d} \log F_{v}^{\mathrm{sync}}}{\mathrm{d} \log F_{v}^{\mathrm{hadr}}}=\frac{\frac{s+1}{2}(1+\beta)-\alpha_{\mathrm{X}}}{2-(1-\beta)\left(\alpha_{\gamma}+2-s\right)}$,

so that for $s=2$ and the observed spectral indices, one would expect the slope of the $\log \left(F_{\mathrm{X}}\right)$ versus $\log \left(F_{\gamma}\right)$ to be $(0.18+$ $1.5 \beta) /(0.91+1.09 \beta)$. This remains lower than 1 .

\subsection{Spatial comparison}

The main difficulty of a leptonic model to account for the observations in RX J1713.7-3946 is that it requires a weak magnetic field (on the order of $10 \mu \mathrm{G}$; AH06) to explain the rather high $\gamma$ to X-ray ratio, if the emitting volume is the same. This is inconsistent with the magnetic field derived from the width of the $\mathrm{X}$-ray filaments ( $70 \mu \mathrm{G}$ or so). A possible reason, suggested by Lazendic et al. (2004), is that the magnetic turbulence decays behind the shock faster than the electrons lose energy. This leaves a larger volume (downstream) to inverse Compton than synchrotron, and does not require that large a magnetic field to begin with. A definite prediction is then that the $\gamma$-ray emission should peak inside the X-rays.

When comparing the $\mathrm{X}$ - and $\gamma$-ray radial profiles (see Sect. 4.3.2) we did see a radial shift, particularly visible in region 6 (West), between the X-and $\gamma$-ray emission. However, the shift is in the opposite direction i.e., the X-ray emission peaks at smaller radius than the $\gamma$-ray emission. The value of this shift for region 6 is $\sim 0.06^{\circ}=1.2 \mathrm{pc}$ for a remnant at $1 \mathrm{kpc}$. Another effect that we have not discussed is that because of the magnetic jump at the shock (typically a factor of 3 if the magnetic field is mostly turbulent and isotropic), the synchrotron emission is strongly suppressed ahead of the shock, whereas the inverse Compton emission will decrease more smoothly over one diffusion length. In a Bohm regime, the diffusion coefficient is $D_{\mathrm{B}}=r_{\mathrm{L}} c / 3$, where $r_{\mathrm{L}}$ is the Larmor radius, and the diffusion length $l_{\text {diff }}=D_{\mathrm{B}} / V_{\mathrm{sh}}$, where $V_{\mathrm{sh}}$ is the shock wave velocity. For an electron energy of $16 \mathrm{TeV}$, a shock speed of $4000 \mathrm{~km} \mathrm{~s}^{-1}$ and an upstream magnetic field of $10 \mu \mathrm{G}$, we have $l_{\text {diff }}=0.1 \mathrm{pc}$. This is small in comparison with the shift of $1.2 \mathrm{pc}$.

However there is another purely geometric explanation of why the X-rays peak inside the $\gamma$-rays. From Fig. 10 the effect is significant only in regions 6 (West) and 8 (North). In both regions, it looks from the image (Fig. 1) that the remnant extends beyond the main X-ray peak. This is typical of a line of sight superposition of higher density (brighter, slower shock) and a region of lower density (fainter, faster shock). This idea is supported by the observations in CO (Moriguchi et al. 2005) showing that clouds are present in those particular regions. From Fig. 7, we know that the X-ray brightness increases much faster than the $\gamma$-ray brightness. The X-ray radial profile is then dominated by the inner bright edge, whereas the outer plateau may contribute significantly to the $\gamma$-ray emission and shift the 
integral along the line of sight to a larger radius. Because of that possibility, we cannot say that the radial profile is incompatible with a leptonic model.

In a hadronic model, the simplest way of determining whether the $\gamma$-ray emission is further out is to ascertain whether a sizable fraction of the flux comes from outside the shock. This is possible and expected (Malkov et al. 2005) if the ambient density increases ahead of the shock (SNR entering a molecular cloud). There are then vast amounts of upstream gas to be used as targets for accelerated protons. A definite prediction of that model is that the remnant should look larger as energy increases because it will be governed by the diffusion length. The energy range in the HESS data may be enough to test that, and certainly will after HESS 2.

\section{Summary}

New XMM-Newton observations of RX J1713.7-3946 have allowed us to complete the coverage of the remnant. We have then carried out a comparison of the remnant in X-and $\gamma$-rays. The radio emission has also been studied. This has led us to the following conclusions:

1. The small-scale variations in the X-ray photon index are largely smeared out at the resolution of the HESS telescope. This is consistent with the non detection of photon index variation in the $\gamma$-rays.

2. The mean X-ray photon index (of value 2.32) is slightly steeper than the $\gamma$-ray one (2.09). This is expected in a leptonic scenario, as the electrons emitting $\gamma$-ray inverse Compton have a lower energy than those emitting X-ray synchrotron. The photon index offset is thus the result of the spectrum cutoff of the electrons.

3. The comparison between the X-ray and $\gamma$-ray integrated flux suggests a non-linear correlation with $\mathrm{d} \log F_{v}^{\mathrm{X}} / \mathrm{d} \log F_{v}^{\gamma}=$ $2.41 \pm 0.55$. If the range of flux that we see is caused by the variation in the density around the remnant, then a leptonic model can more easily reproduce the observed $\mathrm{X} / \gamma$-ray correlation.

4. The comparison between the radial profiles in X-and $\gamma$-rays indicates that for some regions, the $\mathrm{X}$-ray emission comes more from the inside of the remnant than in $\gamma$-rays. This can be explained in a hadronic model if a fraction of the $\gamma$-ray flux originates in the outside of the remnant (interaction of the remnant with a cloud). However this radial shift could also be due to superposition effects. Therefore, we cannot rule out the leptonic scenario.

5. Concerning the radio counterpart of the remnant, we have shown that one of the brightest arcs seen at $1.4 \mathrm{GHz}$ (Arc 2) is of thermal origin and probably not associated with the remnant. Taking this into account, we have derived a lower limit to the integrated flux density of $22 \mathrm{Jy}$ and an upper limit of $26 \mathrm{Jy}$.

Acknowledgements. This project was partially funded by ECOS-SUD FranceArgentina A04U03 program and ANPCYT, CONICET and UBACYT (Argentina) grants. G. Dubner and E. Giacani are members of CONICET (Argentina). M. Ortega is a Doctoral Fellow of CONICET (Argentina).

\section{Appendix A: Correlation between the X-ray and $\gamma$-ray fluxes}

The synchrotron emission can be written

$F_{v}^{\text {sync }} \propto K_{\mathrm{e}} B^{\frac{s+1}{2}} v^{-\frac{s-1}{2}} S\left(\frac{v}{v_{\mathrm{c}}^{\text {sync }}}\right)$ where $S$ is a function characteristic of synchrotron emission and the shape of the electron cutoff and $v_{\mathrm{c}}^{\text {sync }} \propto B E_{\mathrm{c}, \mathrm{e}}^{2} \propto V_{\mathrm{sh}}^{2}$. Then

$\frac{\mathrm{d} \log F_{v}^{\text {sync }}}{\mathrm{d} \log n}=1+\frac{s+1}{2} \beta-\frac{S^{\prime}}{S} \frac{v}{v_{\mathrm{c}}^{\text {sync }}} \frac{\mathrm{d} \log v_{\mathrm{c}}^{\text {sync }}}{\mathrm{d} \log n}$.

Noting that

$\frac{\mathrm{d} \log F_{v}^{\text {sync }}}{\mathrm{d} \log v}=-\frac{s-1}{2}+\frac{S^{\prime}}{S} \frac{v}{v_{\mathrm{c}}^{\text {sync }}}=-\alpha_{\mathrm{X}}$

is the X-ray spectral slope (in energy, not photons) and that $\mathrm{d} \log v_{\mathrm{c}}^{\text {sync }} / \mathrm{d} \log n=-1$, we can write

$\frac{\mathrm{d} \log F_{v}^{\mathrm{sync}}}{\mathrm{d} \log n}=1+\frac{s+1}{2} \beta-\alpha_{\mathrm{X}}+\frac{s-1}{2}=\frac{s+1}{2}(1+\beta)-\alpha_{\mathrm{X}}$.

As long as the Klein-Nishina reduction of the cross-section is not reached (this is still true for $10 \mathrm{TeV}$ electrons incident on the $\mathrm{CMB}$ ) the inverse Compton emission may be written in the same way as Eq. (A.1) but without any explicit $B$ term and with $v_{\mathrm{c}}^{\mathrm{IC}} \propto E_{\mathrm{c}, \mathrm{e}}^{2} \propto V_{\mathrm{sh}}^{2} / B$. The same line of reasoning then leads to

$\frac{\mathrm{d} \log F_{v}^{\mathrm{IC}}}{\mathrm{d} \log n}=1-(1+\beta)\left(\alpha_{\gamma}-\frac{s-1}{2}\right)$

in which $\alpha_{\gamma}$ is the $\gamma$-ray spectral slope.

Interpreting Eqs. (A.4) and (A.5) together, we then predict a flux-flux correlation comparable that in Fig. 9

$\frac{\mathrm{d} \log F_{v}^{\mathrm{sync}}}{\mathrm{d} \log F_{v}^{\mathrm{IC}}}=\frac{\frac{s+1}{2}(1+\beta)-\alpha_{\mathrm{X}}}{1-(1+\beta)\left(\alpha_{\gamma}-\frac{s-1}{2}\right)}$.

If we now turn to the hadronic hypothesis, the $\pi^{0}$ decay emission may be written

$F_{v}^{\text {hadr }} \propto K_{\mathrm{p}} n v^{1-s} H\left(\frac{v}{v_{\mathrm{c}}^{\text {hadr }}}\right)$

where $H$ is a function characteristic of $\pi^{0}$ emission and the shape of the proton cutoff, and $v_{\mathrm{c}}^{\text {hadr }} \propto E_{\mathrm{c}, \mathrm{p}} \propto B V_{\mathrm{sh}}^{2}$. The same line of reasoning then leads to

$\frac{\mathrm{d} \log F_{v}^{\mathrm{hadr}}}{\mathrm{d} \log n}=2-(1-\beta)\left(\alpha_{\gamma}+1-s\right)$

in which we now assume that hadronic emission dominates the $\gamma$-ray spectral slope. Putting together Eqs. (A.4) and (A.8), we then predict the flux-flux correlation in the hadronic model to be

$\frac{\mathrm{d} \log F_{v}^{\mathrm{sync}}}{\mathrm{d} \log F_{v}^{\mathrm{hadr}}}=\frac{\frac{s+1}{2}(1+\beta)-\alpha_{\mathrm{X}}}{2-(1-\beta)\left(\alpha_{\gamma}+1-s\right)}$.

There remains the possibility (Malkov et al. 2005) that the density increases rapidly outwards (SNR hitting a shell) to the point where most of the $\gamma$-ray emission is produced outside the remnant (in the precursor). In that case the width of the precursor increases in proportion to $E$, so that the spectral shape of the $\gamma$-ray emission may be estimated by multiplying the terms in Eq. (A.7) by $E$ (Zirakashvili \& Aharonian 2007). This in turn changes Eq. (A.8) into

$\frac{\mathrm{d} \log F_{v}^{\mathrm{hadr}}}{\mathrm{d} \log n}=2-(1-\beta)\left(\alpha_{\gamma}+2-s\right)$

and changes Eq. (A.9) into

$\frac{\mathrm{d} \log F_{v}^{\mathrm{sync}}}{\mathrm{d} \log F_{v}^{\mathrm{hadr}}}=\frac{\frac{s+1}{2}(1+\beta)-\alpha_{\mathrm{X}}}{2-(1-\beta)\left(\alpha_{\gamma}+2-s\right)}$ 


\section{Appendix B: Detailed calculation for electrons dominated by radiative losses}

If the cutoff in the electron spectrum is defined by synchrotron cooling as we assume in Sect. 5.2.2 and Appendix A, then the cooling continues downstream and the electron distribution integrated over space becomes steeper (the power law index increases by 1) down to a break energy $E_{\mathrm{b}} \propto B^{-2} t_{0}^{-1}$ in which $t_{0}$ is the SNR age (Zirakashvili \& Aharonian 2007). Since we are interested only in what happens above $E_{\mathrm{b}}$, this can be accounted for in Eq. (A.1) by changing $s$ to the steeper value and considering that the normalization $K_{\mathrm{e}}$ follows $E_{\mathrm{b}}$ (the total number of electrons is always dominated by those below $\left.E_{\mathrm{b}}\right)$ or $B^{-2}\left(t_{0}\right.$ is the same in all parts of the SNR). This amounts to adding $-2 \beta$ to Eqs. (A.4) and (A.5), resulting in

$\frac{\mathrm{d} \log F_{v}^{\mathrm{sync}}}{\mathrm{d} \log n}=\frac{s+1}{2}-\alpha_{\mathrm{X}}+\frac{s-3}{2} \beta$, and
$\frac{\mathrm{d} \log F_{v}^{\mathrm{IC}}}{\mathrm{d} \log n}=\frac{s+1}{2}-\alpha_{\gamma}-\beta\left(\alpha_{\gamma}+\frac{5-s}{2}\right)$.

For the most interesting case $s=3$, we then have

$\frac{\mathrm{d} \log F_{v}^{\mathrm{sync}}}{\mathrm{d} \log F_{v}^{\mathrm{IC}}}=\frac{2-\alpha_{\mathrm{X}}}{2-\alpha_{\gamma}-\beta\left(\alpha_{\gamma}+1\right)}$.

For $\alpha_{\mathrm{X}}=1.32$ and $\alpha_{\gamma}=1.09$, we obtain $0.68 /(0.91-2.09 \beta)$. This is very different from what is obtained with $s=2$ and no break in the electron spectrum, but reaches the observed value of 2.41 for a very similar value of $\beta=0.30$.

The pion decay emission is insensitive to synchrotron cooling so that a "radiative" model would predict $\mathrm{d} \log F_{v}^{\text {sync }} / \mathrm{d} \log F_{v}^{\text {hadr }}=0.68 /(1.91+0.09 \beta)$, which is again always less than 1 .

The same framework also naturally predicts variations in the spectral index that are in accordance with the flux variations, the computation of which requires a specific representation of the spectral shape. Suitable approximate formulae are given by Zirakashvili \& Aharonian (2007) in their Eqs. (35) and (46). They apply when there is no discontinuity in the magnetic field at the shock. This is not the most likely situation in our opinion, but it is still interesting to carry out the exercise. The observed average spectral slope in X-rays $\alpha_{\mathrm{X}}=1.32$ corresponds to $x=v / v_{\mathrm{c}}^{\text {sync }}=4.5$, which agrees with that derived by Tanaka et al. (2008) $\left(v_{\mathrm{c}}^{\text {sync }}=0.67 \mathrm{keV}\right)$. In the same way, the observed spectral slope in $\gamma$-rays $\alpha_{\gamma}=1.09$ corresponds to $y=v / v_{\mathrm{c}}^{\mathrm{IC}}=4.1$. From Eqs. (34) and (45) of Zirakashvili \& Aharonian (2007), we note that $v_{\mathrm{c}}^{\text {IC }} / \nu_{\mathrm{c}}^{\text {sync }}=(1.2 \mathrm{TeV}) /(2.2 \mathrm{keV})$ $(B / 100 \mu \mathrm{G})^{-1}$. For $B \simeq 70 \mu \mathrm{G}$ and $\nu_{\mathrm{c}}^{\text {sync }}=0.67 \mathrm{keV}$, this predicts $v_{\mathrm{c}}^{\mathrm{IC}} \simeq 0.5 \mathrm{TeV}$, which is reasonable.

Returning to the spectral index variations, since $\mathrm{d} \log v_{\mathrm{c}}^{\text {sync }} / \mathrm{d} \log n=-1$ when it is limited by cooling, we have

$\frac{\mathrm{d} \alpha_{\mathrm{X}}}{\mathrm{d} \log n}=-\frac{\mathrm{d} \alpha_{\mathrm{X}}}{\mathrm{d} \log x} \frac{\mathrm{d} \log v_{\mathrm{c}}^{\text {sync }}}{\mathrm{d} \log n}=x \frac{\mathrm{d} \alpha_{\mathrm{X}}}{\mathrm{d} x}$, and $\frac{\mathrm{d} \alpha_{\mathrm{X}}}{\mathrm{d} \log n}=0.25 x^{0.5}-0.3795 x^{0.6}\left(1+0.46 x^{0.6}\right)^{-2}$.

At $x=3.5$, this infers that $\mathrm{d} \alpha_{\mathrm{X}} / \mathrm{d} \log n=0.32$. It then predicts that $\mathrm{d} \log F_{v}^{\text {sync }} / \mathrm{d} \alpha_{\mathrm{X}}=0.74 / 0.32=2.3$. The peak-to-peak dispersion that is observed in the X-ray flux $\Delta \log F_{v}^{\text {sync }} \simeq 1.2$ should then be associated with a peak-to-peak dispersion in the slope $\Delta \alpha_{\mathrm{X}}=0.53$. This is larger than what is observed in Fig. 8 $\left(\Delta \alpha_{X}=0.27\right)$. This is qualitatively expected because flux variations are also affected by the geometry (the regions do not cover the same fraction of the SNR). However, the small amplitude of the index variations implies that the fraction of the flux variations that can be attributed to geometry $\Delta \log F_{v}^{\text {geom }}$ is large. It should add quadratically with the part due to density variations $\left(2.3 \times \Delta \alpha_{\mathrm{X}}\right)$, so that $\Delta \log F_{v}^{\text {geom }} \simeq 1.17$. Geometrical effects should of course affect the $\gamma$-ray emission in the same way, but the observed dispersion in $\gamma$-ray flux is then only $\Delta \log F_{v}^{\text {sync }} \simeq 0.6$. This means we are at a dead-end. This specific model cannot explain at the same time the relatively uniform X-ray spectra and the larger contrast between the X-ray fluxes than the $\gamma$-ray fluxes.

\section{References}

Aharonian, F. A., Akhperjanian, A. G., Aye, K.-M., et al. 2004, Nature, 432, 75 Aharonian, F., Akhperjanian, A. G., Bazer-Bachi, A. R., et al. 2006, A\&A, 449, 223 (AH06)

Arnaud, M., Neumann, D. M., Aghanim, N., et al. 2001, A\&A, 365, L80

Ballet, J. 2006, Adv. Space Res., 37, 1902

Benjamin, R. A., Churchwell, E., Babler, B. L., et al. 2003, PASP, 115, 953

Berezhko, E. G., \& Völk, H. J. 2006, A\&A, 451, 981

Carter, J. A., \& Read, A. M. 2007, A\&A, 464, 1155

Cassam-Chenaï, G., Decourchelle, A., Ballet, J., et al. 2004, A\&A, 427, 199

Conti, P. S., \& Crowther, P. A. 2004, MNRAS, 355, 899

Ellison, D. C., Slane, P., \& Gaensler, B. M. 2001, ApJ, 563, 191

Enomoto, R., Tanimori, T., Naito, T., et al. 2002, Nature, 416, 823

Fukui, Y., Moriguchi, Y., Tamura, K., et al. 2003, PASJ, 55, L61

Hiraga, J. S., Uchiyama, Y., Takahashi, T., \& Aharonian, F. A. 2005, A\&A, 431, 953

Koyama, K., Kinugasa, K., Matsuzaki, K., et al. 1997, PASJ, 49, L7

Lazendic, J. S., Slane, P. O., Gaensler, B. M., et al. 2003, ApJ, 593, L27

Lazendic, J. S., Slane, P. O., Gaensler, B. M., et al. 2004, ApJ, 602, 271

Malkov, M. A., Diamond, P. H., \& Sagdeev, R. Z. 2005, ApJ, 624, L37

McClure-Griffiths, N. M., Dickey, J. M., Gaensler, B. M., et al. 2005, ApJS, 158, 178

Moriguchi, Y., Tamura, K., Tawara, Y., et al. 2005, ApJ, 631, 947

Muraishi, H., Tanimori, T., Yanagita, S., et al. 2000, A\&A, 354, L57

Pannuti, T. G., Allen, G. E., Houck, J. C., \& Sturner, S. J. 2003, ApJ, 593, 377

Pfeffermann, E. \& Aschenbach, B. 1996, in Roentgenstrahlung from the Universe, ed. H. U. Zimmermann, J. Trümper, \& H. Yorke, 267

Pratt, G. W., \& Arnaud, M. 2002, A\&A, 394, 375

Reach, W. T., Rho, J., Tappe, A., et al. 2006, AJ, 131, 1479

Rothenflug, R., Ballet, J., Dubner, G., et al. 2004, A\&A, 425, 121

Russeil, D. 2003, A\&A, 397, 133

Slane, P., Gaensler, B. M., Dame, T. M., et al. 1999, ApJ, 525, 357

Tanaka, T., Uchiyama, Y., Aharonian, F. A., et al. 2008, ApJ, 685, 988

Testori, J. C., Reich, P., Bava, J. A., et al. 2001, A\&A, 368, 1123

Wang, Z. R., Qu, Q. Y., \& Chen, Y. 1997, A\&A, 318, L59

Zirakashvili, V. N., \& Aharonian, F. 2007, A\&A, 465, 695 